\title{
Development of Visual Feedback Training Using Functional Electrical Stimulation Therapy for Balance Rehabilitation
}

AUTHORS:

Kelvin Chow ${ }^{1}$, Emerson P. Grabke², Jae Lee ${ }^{2}$, Jaeeun Yoo $^{2}$, Kristin E. Musselman²,4 Kei Masani²,4.

\section{INSTITUTION:}

1. Department of Mechanical and Industrial Engineering, University of Toronto.

2. Institute of Biomaterials and Biomedical Engineering, University of Toronto.

3. Department of Physical Therapy, Faculty of Medicine, University of Toronto.

4. Toronto Rehabilitation Institute, University Health Network.

\section{EDITOR'S NOTE:}

The following work was recognized as a winning project (specifically third place) at the Undergraduate Engineering Research Day 2017 (UnERD 2017), and was recommended for publication (as a graphical abstract) within the STEM Fellowship Journal. UnERD is an undergraduate research competition, held annually at the University of Toronto.

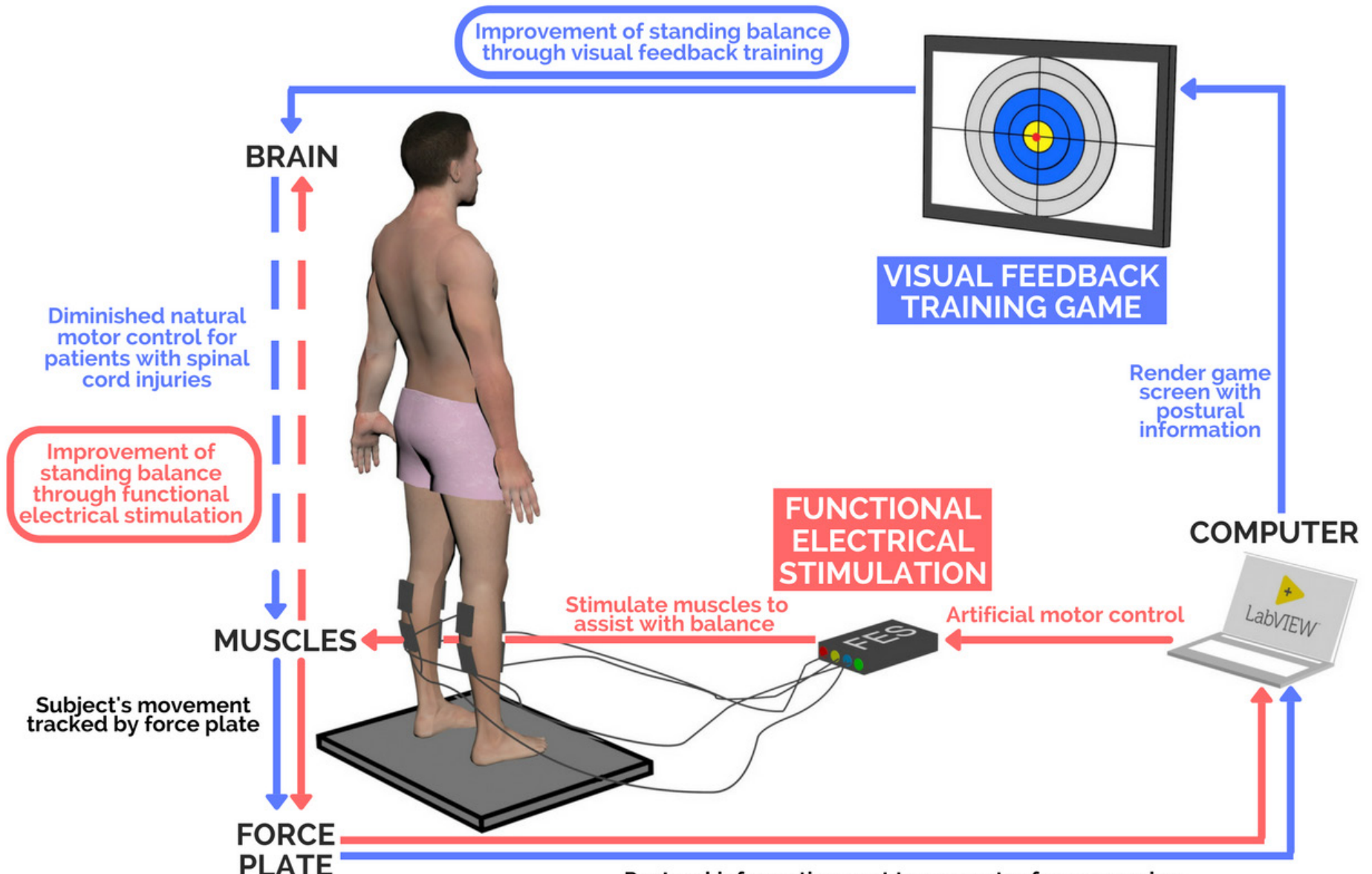

PLATE

Postural information sent to computer for processing

VISUAL FEEDBACK

FUNCTIONAL ELECTRICAL STIMULATION

NOVEL BALANCE 


\section{Abstract}

Individuals with incomplete spinal cord injuries (iSCl) often experience impairments in motor control of lower-limb muscles, causing a lack of balance when standing. These individuals are usually heavily dependent on others, reducing their self-sufficiency and quality of life. Our previous study has shown that visual feedback training (VFT) of tracking an individual's center of pressure (COP) can improve balance stability of those with iSCI (1). Another of our previous studies demonstrated that functional electrical stimulation (FES) augments therapeutic effects in general (2-8). Thus, VFT in combination with FES is hypothesized to improve the standing balance ability of individuals with iSCI more effectively than a VFT or FES system. The purpose of this study was to develop a novel therapeutic system by combining VFT with FES.

We developed this system by utilizing LabVIEW (17.0, National Instruments Corp., Austin, USA). The system hardware consisted of a force plate, an electrical stimulator, and a computer. The COP was calculated using the force plate signals, which was displayed in the monitor in front of the participant. We developed four COP-based games, each forcing the participant to shift their COP in various movement patterns. FES will provide electrical stimulation to participant plantarflexors and dorsiflexors to assist with movements, in which stimulus intensity will be modulated through a proportionalderivative $(\mathrm{PD})$ feedback controller that mimics the physiological control system for standing $(9,10)$.

In this study, we tested the practicality of our VFT games with

\section{References}

1. Sayenko DG, Alekhina MI, Masani K, Vette AH, Obata H, Popovic MR, et al. Positive effect of balance training with visual feedback on standing balance abilities in people with incomplete spinal cord injury. Spinal Cord. 2010;48:886-93.

2. Popovic MR, Masani K, Micera S. Functional electrical stimulation therapy: Recovery of function following spinal cord injury and stroke, In: Reinkensmeyer D, Dietz V. Neurorehabilitation Technology. Springer. c2012. p105-21.

3. Popovic MR, Kapadia N, Zivanovic V, Furlan JC, Craven BC, McGillivray C. Functional electrical stimulation therapy of voluntary grasping versus only conventional rehabilitation for patients with subacute incomplete tetraplegia: a randomized clinical trial. Neurorehabilitation and Neural Repair. 2011; 25:433-42.

4. Vette AH, Masani K, Popovic MR. Implementation of a physiologically identified PD feedback controller for regulating the active ankle torque during quiet stance. IEEE Trans. Neural Syst. Rehabil. Eng. 2007;15:235-43.

5. Vette AH, Masani K, Kim JY, Popovic MR. Closed-loop control of functional electrical stimulation-assisted arm-free standing in individuals with spinal cord injury: a feasibility study. Neuromodulation. 2009;12:22-32. seven able-bodied participants (age 19-24 years). Each participant performed a balance assessment before playing the VFT games to determine their balance ability. Our focus was on developing effective VFT games and assessing each participant's balance ability against their VFT game scores. Three out of four games showed a significant bivariate correlation between balance ability and VFT scores ( $r>0.754$ for $n=7$ ), indicating that the games were appropriately designed. The validity of the FES controller was demonstrated in another study to be effective. The parameters of the PD controller were optimized so that the stimulation intensity would match the natural muscle contraction of the plantarflexors and dorsiflexors, acquired through electromyography (EMG) signals.

It is expected that rehabilitation combining VFT with FES will improve static and dynamic balance stability in a more effective way than isolated therapy techniques. Since this study has shown the validity of our VFT games, we would like to integrate the VFT and FES systems and run clinical trials on patients with iSCl, with the goal of improving their balance ability in the future. Through visual feedback training, the brain improves spatial awareness by visualizing their body displacement and orientation. Applying stimulation to the muscles re-trains the central nervous system and, over time, allows patients with iSCI to partially regain motor control. Thus, the proposed system will help individuals with iSCI improve their balance ability and increase their quality of life.

\section{Keywords}

Spinal Cord Injury, Rehabilitation, Standing, Visual Feedback Training, and Functional Electrical Stimulation
6. Vette AH, Masani K, Nakazawa K, Popovic MR. Neural-mechanical feedback control scheme generates physiological ankle torque fluctuation during quiet stance. IEEE Trans. Neural Syst. Rehabil. Eng. 18 2010;18:86-95.

7. Tan JF, Masani K, Vette AH, Zariffa J, Robinson M, Lynch C, et al. Inverted pendulum standing apparatus for investigating closed-loop control of ankle joint muscle contractions during functional electrical stimulation. International Scholarly Research Notices. 2014:ID192097.

8. Same MB, Rouhani H, Masani K, Popovic MR. Closed-loop control of ankle plantarflexors and dorsiflexors using an inverted pendulum apparatus: a pilot study. Journal of Automatic Control. 2013;21:31-36.

9. Masani K, Vette AH, Popovic MR. Controlling balance during quiet standing: proportional and derivative controller generates preceding motor command to body sway position observed in experiments. Gait \& Posture. 2006; 23:164-72.

10. Masani K, Vette AH, Kawashima N, Popovic MR. Neuromusculoskeletal torque-generation process has a large destabilizing effect on the control mechanism of quiet standing. J Neurophysiol. 2008;100:1465-75. 